\title{
FOURIER-STIELTJES TRANSFORMS OF MEASURES ON INDEPENDENT SETS
}

\author{
BY WALTER RUDIN ${ }^{1}$ \\ Communicated March 30, 1960
}

A subset $E$ of the real line $R$ will be called independent if the following is true: for every choice of distinct points $x_{1}, \cdots, x_{k}$ in $E$ and of integers $n_{1}, \cdots, n_{k}$, not all 0 , we have $n_{1} x_{1}+\cdots+n_{k} x_{k} \neq 0$. The main result of this note is

THEOREM I. There exists an independent, compact, perfect set $Q$ in $R$ which carries a positive measure $\sigma$ whose Fourier-Stieltjes transform

$$
\int_{-\infty}^{\infty} e^{i x y} d \sigma(x) \quad(y \in R)
$$

tends to 0 as $|y| \rightarrow \infty$.

Sketch of proof. It is known ([5, Theorem IV] and [6, p. 25]) that there is a compact perfect set $P$ in $R$ which is not a basis (i.e., the set of all finite sums $\sum n_{i} x_{i}$, with $x_{i} \in P$ and integers $n_{i}$, does not cover $R$ and hence has measure 0 ) but which carries a positive measure $\mu$ whose F.S. transform vanishes at infinity. A certain deformation of $P$ will yield our set $Q$.

$P$ is constructed as the intersection of a sequence of sets $E_{r}$ which are unions of $2^{r}$ disjoint intervals $I_{j, r}$. Set $P_{j, r}=P \cap I_{j, r}$, for $1 \leqq j \leqq 2^{r}$.

REMARK 1. Since $P$ is not a basis, the set of all points $w=\left(w_{1}, \cdots, w_{k}\right)$ in $R^{k}$ such that $\sum_{1}^{k} n_{j}\left(x_{j}+w_{j}\right)=0$ for some choice of $x_{1}, \cdots, x_{k}$ in $P$ is, for each choice of integers $n_{1}, \cdots, n_{k}$, a closed set of measure 0 (a union of certain hyperplanes).

REMARK 2. Since there exists a function in $L^{1}(R)$ whose Fourier transform is 1 on $P_{j, r}$ and is 0 on the rest of $P$, we have

$$
\lim _{|y| \rightarrow \infty} \int_{P_{j, r}} e^{i x y} d \mu(x)=0 \quad\left(1 \leqq j \leqq 2^{r}\right) .
$$

Choose a sequence $\left\{c_{r}\right\}, 0<c_{r}<1$, such that $\prod_{0}^{\infty} c_{r}>0$. Put $f_{0}(x)$ $=x$, and inductively define a sequence of functions $f_{r}$ on $P$, of the form

$$
f_{r}(x)=x+w_{j, r} \quad\left(x \in P_{j, r}\right) .
$$

Assume $f_{r}$ is constructed, and has the property that the condition

\footnotetext{
${ }^{1}$ Research Fellow of the Alfred P. Sloan Foundation.
} 


$$
0<\sum_{1}^{2^{r}}\left|n_{j}\right|, \quad\left|n_{j}\right| \leqq r, \quad x_{j} \in P_{j, r}
$$

implies

$$
\sum_{1}^{2^{r}} n_{j} f_{r}\left(x_{j}\right) \neq 0
$$

By Remark 1 we can construct $f_{r+1}$ so that $\left(\mathrm{A}_{r+1}\right)$ implies $\left(\mathrm{B}_{r+1}\right)$ and so that $\left(\mathrm{A}_{r}\right)$ implies

$$
\left|\sum_{1}^{2^{r}} n_{j} f_{r+1}\left(x_{j}\right)\right|>c_{r}\left|\sum_{1}^{2^{r}} n_{j} f_{r}\left(x_{j}\right)\right| .
$$

Remark 2 implies that the functions

$$
g_{r}(y)=\int_{P} \exp \left\{i f_{r}(x) y\right\} d \mu(x) \quad(r=0,1,2, \cdots),
$$

vanish at infinity, and it follows (again from Remark 1) that we can subject $f_{r+1}$ to the further requirements that $\left|f_{r+1}(x)-f_{r}(x)\right|<2^{-r}$ for $x \in P$ and that $\left|g_{r+1}(y)-g_{r}(y)\right|<2^{-r}$ for all real $y$.

Define $f(x)=\lim _{r \rightarrow \infty} f_{r}(x)$. Our construction shows that no finite sum $\sum n_{j} f\left(x_{j}\right)$ can be 0 if the $x_{j}$ are distinct points of $P$ and the $n_{j}$ are integers, not all 0 . It follows that $f$ is a homeomorphism of $P$ onto an independent perfect set $Q$. Since the sequence $\left\{g_{r}\right\}$ converges uniformly, we have

$$
\lim _{|y| \rightarrow \infty} \int_{P} e^{i f(x) y} d \mu(x)=0 .
$$

The formula $\sigma(f(E))=\mu(E)$ defines a measure $\sigma$ on $Q$, such that

$$
\int_{P} e^{i f(x) y} d \mu(x)=\int_{Q} e^{i t y} d \mu(t),
$$

and the theorem follows from (4).

We now list some consequences.

1. Let $M$ be the Banach algebra of all bounded Borel measures on $R$, with convolution as multiplication, and let $M_{0}$ be the algebra of all $\mu \in M$ whose F.S. transforms vanish at infinity. It is known (see [4] for references) that $M$ is not symmetric. Theorem I implies

THEOREM II. $M_{0}$ is not symmetric. ${ }^{2}$

2 This answers a question raised by Irving Glicksberg. 
This is proved from Theorem I by showing (either by Sreider's original method [6, pp. 21-22] or by a device due to J. H. Williamson $\left[4\right.$, p. 234]) that there is a $\mu \in M_{0}$ such that the complex conjugate of its Gelfand transform (see [4]) is not the Gelfand transform of any member of $M$.

2. Call a compact set $E$ in $R$ a Helson set if every continuous function on $E$ is the restriction to $E$ of a F.S. transform. There exist perfect Helson sets [3] and every countable, independent, compact set is a Helson set. However, by [1] Theorem I implies

TheOREM III. The independent perfect set $Q$ is not a Helson set.

It follows [3] that there is a bounded function whose spectrum lies in $Q$ but which is not a F.S. transform; i.e., $Q$ carries a "true pseudo-measure," in the terminology of [3].

3. Call a compact set $E$ in $R$ strongly independent if to every continuous function $f$ on $E$, with $|f| \equiv 1$, and to every $\epsilon>0$ there exists $y \in R$ such that $\left|f(x)-e^{i y x}\right|<\epsilon$ for all $x \in E$. This definition stems from Kronecker's theorem: every finite independent set is strongly independent.

Hewitt and Kakutani [2] have constructed strongly independent perfect sets. It is not hard to show that strongly independent sets are Helson sets, and we conclude:

THEOREM IV. The independent perfect set $Q$ is not strongly independent.

4. Finally, we point out that $Q$ furnishes an example of an independent perfect set which is a set of multiplicity (even in the restricted sense; see [7, pp. 344, 348]) for the convergence of trigonometric series, and that it is not a set of type $N[7, \mathrm{p} .236]$, whereas every strongly independent set is of type $N$. In fact, to every strongly independent set $E$ one can associate an increasing sequence of integers $n_{k}$ such that $\sum \sin n_{k} x$ converges absolutely for all $x \in E$.

\section{REFERENCES}

1. Henry Helson, Fourier transforms on perfect sets, Studia Math. vol. 14 (1954) pp. 209-213.

2. Edwin Hewitt and Shizuo Kakutani, $A$ class of multiplicative linear functionals on the measure algebra of a locally compact abelian group, mimeographed.

3. Jean-Pierre Kahane and Raphael Salem, Sur les ensembles linéaires ne portant pas de pseudo-mesures, C. R. Acad. Sci. Paris vol. 243 (1956) pp. 1185-1187.

4. Walter Rudin, Measure algebras on abelian groups, Bull. Amer. Math. Soc. vol. 65 (1959) pp. 227-247. 
5. Raphael Salem, On sets of multiplicity for trigonometrical series, Amer. J. Math. vol. 64 (1942) pp. 531-538.

6. Yu. A. Sreider, The structure of maximal ideals in rings of measures with convolution, Amer. Math. Soc. Translation, no. 81, Providence, 1953.

7. Antoni Zygmund, Trigonometric series, 2d ed., vol. I, Cambridge University Press, 1959.

UNIVERSITY OF WISCONSIN

\section{ARITHMETIC PROPERTIES OF CERTAIN POLYNOMIAL SEQUENCES}

BY L. CARLITZ

Communicated by G. B. Huff, March 23, 1960

Consider the sequence of polynomials $\left\{u_{n}(x)\right\}$ that satisfy the recurrence

$$
u_{n+1}(x)=(x+a(n)) u_{n}(x)+b(n) u_{n-1}(x),
$$

where $a(n), b(n)$ are polynomials in $n$ (and possibly some additional indeterminates) with integral coefficients. Moreover it is assumed that

$$
u_{0}(x)=1, \quad u_{1}(x)=a(0), \quad b(0)=0 .
$$

The sequence $\left\{u_{n}(x)\right\}$ is uniquely determined by (1) and (2).

The writer [1, Theorem 1] has proved that if $m \geqq 1, r \geqq 1$, then $u_{n}(x)$ satisfies the congruence

$$
\sum_{s=0}^{r}(-1)^{s}\left(\begin{array}{l}
r \\
s
\end{array}\right) u_{n+s m}(x) u_{(r-s) m}(x) \equiv 0\left(\bmod m^{r_{1}}\right),
$$

for all $n \geqq 1$, where

$$
r_{1}=[(r+1) / 2],
$$

the greatest integer $\leqq(r+1) / 2$. In the present paper it is proved that $u_{n}(x)$ satisfies the simpler congruence

$$
\sum_{s=0}^{r}(-1)^{s}\left(\begin{array}{l}
r \\
s
\end{array}\right) u_{n+s m}(x) u_{m}^{r-s}(x) \equiv 0\left(\bmod m^{r_{1}}\right),
$$

where again $r_{1}$ is defined by (4). Also it is shown that (5) implies

$$
\sum_{s=0}^{r}(-1)^{s}\left(\begin{array}{l}
r \\
s
\end{array}\right) u_{n+s m}(x) u_{k+(r-s) m}(x) \equiv 0\left(\bmod m^{r_{1}}\right),
$$

